\title{
Modelos animais na síndrome metabólica.
}

\section{Animal models in metabolic syndrome.}

Taíse Fuchs'; Marcelo de Paula loureiro, tCbC-PR; lano Emerson Macedo'; David Nocca²; Marius Nedelcu²; Thaís Andrade COSTA-CASAGRANDE ${ }^{1}$

\section{R E S U M O}

O conhecimento sobre modelos animais para estudo metabólico representa a base da pesquisa nessa área. Este trabalho tem por objetivo revisar os principais modelos animais a serem utilizados no estudo da obesidade e da síndrome metabólica. Para isso, pesquisa no banco de dados Pubmed foi realizada usando as palavras-chave "animal models", "obesity", "metabolic syndrome", e "bariatric surgery". Várias espécies de animais podem ser usadas para o estudo de distúrbios metabólicos, no entanto, os roedores, tanto modelos monogênicos quanto modelos de obesidade induzida por dieta (DIO), são os animais mais utilizados nessa área. Animais monogênicos são a melhor escolha se apenas um aspecto estiver sendo avaliado. Animais DIO tendem a demonstrar melhor a interação entre doença, ambiente e gene. No entanto, eles ainda não são totalmente eficazes para a compreensão de todos os mecanismos dessa doença.

Descritores: Modelos Animais. Obesidade. Síndrome Metabólica. Cirurgia Bariátrica.

\section{INTRODUÇÃO}

A obesidade e a síndrome metabólica estão lentre as principais causas de mortalidade mundial e seus mecanismos patogênicos ainda não estão totalmente compreendidos ${ }^{1}$. Dessa forma, desenvolver novos métodos de investigação das doenças com o intuito de promover a profilaxia, controle ou a cura dessas afecções torna-se uma prioridade. O uso de animais na experimentação foi e continua sendo de grande importância em pesquisas médicas, inclusive para estudo do metabolismo. Porém os resultados obtidos em estudos pré-clínicos não necessariamente são análogos aos encontrados no ser humano². Traduzir os achados em animais para seres humanos pode ser um desafio, tanto pela diferença na fisiologia entre espécies, quanto pela própria falha na adoção do modelo de pesquisa ${ }^{3}$. Sendo assim, a escolha de um modelo válido para estudo de qualquer doença, visando à máxima semelhança com o que ocorre no paciente humano, é fundamental ${ }^{2}$.

Este trabalho tem por objetivo revisar os principais modelos animais utilizados no estudo da obesidade e síndrome metabólica.

\section{MÉTODOS}

Foram pesquisados artigos na base de dados Pubmed, nos últimos dez anos, selecionados por meio das palavras-chave "animal models", "obesity", "metabolic syndrome" e "bariatric surgery".

\section{RESULTADOS}

\section{Modelos de roedores}

Os modelos de roedores mais utilizados para o estudo da obesidade e síndrome metabólica são divididos principalmente em modelos geneticamente modificados, sendo os mais utilizados os animais monogênicos (mutação ligada a um gene apenas), e modelos obesos induzidos por dieta (diet-induced obesity - DIO) $)^{2,4}$.

Modelos monogênicos têm como vantagem o desenvolvimento de afecções mais graves e com fenótipo bem distinto, o que facilita estudos, principalmente de fármacos, já que os efeitos são melhor observados. Além disso, costumam produzir experimentos mais curtos, pois não necessitam de programas longos de alimentação para indução da obesidade $^{5}$. Como a base genética é homogênea e os

1 - Universidade Positivo, Pós-Graduação em Biotecnologia, Curitiba, PR, Brasil. 2 - Université Montpellier, CHU de Montpellier, Montpellier, Hérault, França. 
fatores ambientais são controlados, a variabilidade dos resultados é mínima, possibilitando o uso de amostras menores ${ }^{6}$. Observações derivadas destas linhagens puras, entretanto, podem não ser semelhantes às encontradas na população humana, já que a obesidade é sabidamente uma doença multifatorial. Nesse caso, modelos DIO parecem ser os que mais se aproximam dos mecanismos promotores de obesidade e síndrome metabólica em humanos².

Outra desvantagem dos animais monogênicos é a alta mortalidade devido à cetose em determinadas linhagens, como no caso do camundongo $\mathrm{db} / \mathrm{db}$, além da necessidade de manutenção sofisticada dos animais, o que encarece a pesquisa ${ }^{6}$. Em geral, o custo de um animal monogênico é de no mínimo 100 a 400 dólares, variando com a linhagem escolhida, o que pode ainda aumentar dependendo do sexo, peso e idade escolhidos para a pesquisa. Já o rato Wistar e o Sprague Dawley (SD), modelos DIO mais utilizados, podem ser adquiridos, em média, por 20 dólares cada ${ }^{7,8}$.

\section{Animais geneticamente modificados}

\section{Animais monogênicos}

Um dos animais monogênicos mais utilizados no estudo da obesidade e síndrome metabólica, principalmente o diabetes tipo 2 (DM2), é o camundongo ob/ob9 . A mutação espontânea que leva à obesidade é conhecida desde a década de 50, porém maior ênfase no produto do gene ob, a leptina, foi dada somente a partir de $1994^{4}$. O camundongo ob/ob não produz leptina, hormônio responsável pela saciedade, porém ainda é sensível a ela ${ }^{9}$. A obesidade importante que ocorre nesses animais tem ainda outras causas, como o defeito na termogênese do tecido adiposo marrom, o que leva a uma maior deposição de energia ingerida na forma de gordura10, e lipogênese hepática aumentada ${ }^{11}$.
Este animal apresenta como primeira característica o fenótipo obeso acentuado precoce, caracterizado por hiperfagia 2,4 , seguido de hiperinsulinemia, hiperglicemia moderada e resistência à insulina, além de hipotiroidismo ${ }^{4}$. O animal apresenta esteatose hepática, porém a progressão para hepatite não ocorre. Para isso é necessário a exposição do camundongo a um agente tóxico, diferentemente de humanos, em que a progressão é uma consequência natural da doença ${ }^{12}$.

O tratamento com leptina nestes animais normalmente diminui a ingestão de alimento e aumenta a absorção de glicose em vários tecidos. Além disso, a diminuição crônica do consumo alimentar reduz o peso corporal e melhora a sensibilidade à insulina. A administração de leptina recombinante em humanos obesos por deficiência desse hormônio demonstrou os mesmos efeitos observados nos camundongos. No entanto, a maioria dos indivíduos obesos não apresenta obesidade ocasionada por deficiência na produção de leptina. Ao contrário, este hormônio normalmente está elevado devido a uma resistência à leptina, demonstrando que a fisiologia do animal não reflete completamente a fisiologia humana².

O camundongo $\mathrm{db} / \mathrm{db}$ é fenotipicamente semelhante ao ob/ob, desenvolvendo obesidade rapidamente após o desmame, porém apresenta hiperglicemia mais grave do que o segundo, devido à deficiência no receptor de leptina ${ }^{4}$. O nível glicêmico em animais de sete semanas é, em média, de 700mg/ dl, o que se sustenta por toda sua vida, ao contrário do que ocorre em animais ob/ob, em que os níveis diminuem e normalizam após 12 semanas de idade ${ }^{13}$. Já os níveis de leptina são elevados, já que não há defeito em sua produção ${ }^{14}$. A permanência da hiperglicemia durante toda a vida do animal consiste de vantagem em determinados experimentos em que a idade influencia o resultado. Em estudo objetivando avaliar alterações neurológicas induzidas por hiperinsulinemia e diabetes, camundongos db/ 
$\mathrm{db}$ foram utilizados em diferentes fases de vida (quatro, 14 e 26 semanas de idade) para verificar e correlacionar a progressão da resistência à insulina até a instalação do diabetes clínico e sua evolução. Este modelo animal permitiu aos pesquisadores verificar a progressão da atrofia cerebral decorrente da idade, associada à alteração metabólica ${ }^{15}$.

Apesar dos camundongos $\mathrm{db} / \mathrm{db}$ serem muito utilizados para o estudo do DM2 e suas complicações, como a retinopatia e neuropatia, estes animais não desenvolvem todas as alterações encontradas em humanos, como por exemplo, a deposição amiloide em pâncreas ${ }^{4}$.

O camundongo KK apresenta resistência à insulina e obesidade moderada de origem poligênica. Porém, a introdução da mutação agouti (Ay), considerada um defeito monogênico, produz o modelo KK-Ay, que apresenta o DM2 de forma mais precoce ${ }^{2,16}$. A proteína agouti funciona como um antagonista do receptor de melanocortina-4, o que afeta a regulação energética do organismo, predispondo à obesidade ${ }^{2,17}$. O camundongo KKAy apresenta hiperleptinemia e resistência à leptina sem qualquer defeito no gene ob, assim como, diminuição dos níveis de adiponectina, semelhante ao que ocorre em humanos ${ }^{18}$.

O excesso de peso nesses animais já é observado com dois meses de idade, estabilizando, aos seis meses, em torno de 50 a 60g, com 33\% do peso corporal sendo composto de gordura. O animal tende a apresentar hiperinsulinemia, intolerância à glicose e hiperglicemia, porém, esta normaliza após um ano de idade. Lesão glomerular e glicosúria também foram observadas nesse modelo. Como a obesidade nesse animal ocorre por um aumento do consumo alimentar, a restrição dietética tende a reverter o excesso de peso ${ }^{17}$. Como tanto a obesidade quanto o DM2 aparecem de forma precoce nestes animais, o tempo de experimento acaba por ser mais curto. Estudo utilizando fêmeas KK-Ay com cinco semanas de idade, já obesas e diabéticas, avaliou a administração de composto obtido da geleia real com potencial terapêutico para o DM2 durante quatro semanas. Mesmo em curto prazo, os pesquisadores identificaram a melhora nos níveis glicêmicos e na resistência à insulina ${ }^{16}$.

0 rato Zucker, da mesma forma que o camundongo $\mathrm{db} / \mathrm{db}$, desenvolve a obesidade por apresentar defeito no receptor de leptina, ocasionada por uma mutação no gene $f^{19}$. Esse animal produz leptina, porém, não há atuação do hormônio em seu receptor, levando-o a um estado de hiperfagia, com altos níveis de leptina plasmática. Além da leptina, outros hormônios orexígenos também estão elevados neste modelo ${ }^{20}$. Ratos Zucker adultos apresentam $40 \%$ de seu peso na forma de gordura, assim como, resistência à insulina, porém, a glicemia é normal, sem desenvolvimento evidente do quadro de $\mathrm{DM} 2^{18}$. Esses dados são semelhantes a uma parte da população humana que apresenta obesidade e resistência à

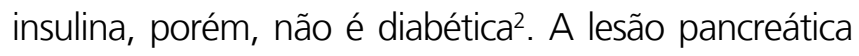
nestes animais, entretanto, não ocorre da mesma forma que em humanos ${ }^{21}$. Este modelo animal é utilizado principalmente para estudos farmacológicos de medicamentos antiobesidade e sensibilizadores de insulina, assim como, de análogos de incretina ${ }^{22}$.

O cruzamento de ratos Zucker consanguíneos acabou desenvolvendo uma subestirpe menos obesa, porém diabética, denominada de rato Zucker obeso diabético (ZDF) ${ }^{23}$. Ratos ZDF machos são mais propensos ao desenvolvimento de DM2, sendo utilizado para seu estudo, assim como, da obesidade, sinalização de leptina ou a interação entre as três alterações ${ }^{5}$.

Também utilizado para o estudo do DM2, o rato Goto-Kakizaki (GK) é um animal não obeso e espontaneamente diabético, obtido pela seleção de ratos Wistar com altos níveis glicêmicos ${ }^{24}$. Animais GK apresentam hiperglicemia em jejum, hiperinsulinemia, intolerância à glicose, já com duas semanas de idade, e início precoce de complicações diabéticas ${ }^{25}$, sendo considerado um dos melhores modelos para estudo dessa doença ${ }^{6}$. 


\section{Animais poligênicos}

Um dos modelos poligênicos utilizados em pesquisas metabólicas é o rato obeso da Nova Zelândia (NZO), animal que desenvolve hiperfagia e obesidade juvenil, mesmo ingerindo dietas com baixo teor de gordura. Além disso, também podem desenvolver $\mathrm{DM} 2^{26}$. Por conta da variação da ocorrência de diabetes, esse modelo foi cruzado com outro animal intolerante à glicose, visando ao desenvolvimento da doença como ocorre em humanos. Apesar das subestirpes desenvolverem a afecção, alguns animais, entretanto, nem sempre apresentam o quadro ${ }^{27}$. Isso demonstra como o desenvolvimento da obesidade e síndrome metabólica é um processo complexo, tanto em animais quanto em humanos, dificultando o entendimento completo de sua patogênese.

A estirpe de roedor JCR:LA é a mais utilizada, por desenvolver, além de aterosclerose, isquemia cardíaca e resistência à insulina ${ }^{27}$. Essa linhagem apresenta obesidade muito mais extrema do que a observada em rato Zucker, assim como, hiperlipidemia grave ${ }^{6}$. Apesar disso, esse modelo possui diferenças significativas na morfologia das lesões ateroscleróticas e não podem demonstrar ainda a mesma patogênese dos humanos ${ }^{28}$.

\section{Animais obesos induzidos por dieta}

A característica mais comum dos animais geneticamente modificados, com exceção do GK, é o início precoce da obesidade. Em seres humanos, no entanto, o ganho de peso pode ocorrer em qualquer idade, sendo mais comum com o avanço da mesma. Além disso, o grau de obesidade em pacientes com DM2 é variável, sendo menos grave em jovens, ao contrário do que ocorre nestes roedores, o que pode afetar muitos aspectos da pesquisa ${ }^{5}$.

Modelos animais DIO são os que mais se aproximam dos mecanismos promotores de obesidade e síndrome metabólica em humanos. Estes animais são normalmente utilizados para estudo do papel da dieta, fisiopatologia e etiologia da doença, além de testes farmacológicos. No entanto, os resultados dos estudos são discrepantes, principalmente em relação à composição das dietas e o tipo de modelo utilizado $^{2,29}$. A dieta moderna em seres humanos é composta normalmente por elevado nível de gorduras e carboidratos. Os estudos com roedores baseiam-se nessas dietas, porém, há variação na quantidade dos componentes utilizados, assim como, em sua fonte, o que pode alterar o fenótipo do animal ${ }^{2}$ e acabar por desenvolver um modelo de obesidade e/ou DM2 com características não padronizadas. Em geral, dietas com altos níveis de frutose imitam a dieta humana e associadas a alto teor de gordura promovem aumento do peso, da gordura abdominal, hiperglicemia e hiperinsulinemia em camundongos ${ }^{30}$. A frutose parece ser importante no desenvolvimento da síndrome metabólica, assim como, da própria obesidade, já que este açúcar leva não só à resistência à insulina, mas também à resistência de leptina, resultando no ganho de peso ${ }^{31-33}$

Geralmente as dietas utilizadas são padronizadas comercialmente, porém existem opções, como a dieta da cafeteria, em que os animais escolhem os alimentos oferecidos. A vantagem dessa dieta é sua alta palatabilidade, além da grande semelhança com a dieta humana, porém, como não é padronizada, o conteúdo nutricional torna-se de difícil avaliação e os animais podem apresentar deficiência de proteínas e hipovitaminose. Apesar de demonstrar ganho de peso importante, essa dieta tende a ser menos utilizada do que as comerciais ${ }^{3}$.

Determinadas linhagens, como a de camundongos S5B/PI ou $\mathrm{A} / \mathrm{J}$ são considerados resistentes à obesidade induzida por dieta, enquanto ratos SD e Wistar desenvolvem essa afecção mais facilmente, o que mostra que a base genética é importante no ganho de peso corporal ${ }^{2}$. A estirpe DIO mais utilizada é de ratos SD, animais 
propensos ao ganho de peso pela dieta desde filhotes, assim como, camundongos C57BL6/J, animais obesos e potencialmente hiperglicêmicos e hiperinsulinêmicos, que desenvolvem obesidade mesmo quando alimentados com dieta padrão ao longo do período de vida do indivíduo, semelhante ao que ocorre em humanos $29,34,35$. Estudo avaliando a eficácia do bypass gástrico em $Y$ de Roux em animais C57BL6/J com esteato-hepatite não alcóolica, confirmou não somente que este animal poderia ser utilizado como modelo da alteração hepática, mas também que a cirurgia poderia conferir modulação da função mitocondrial hepática contribuindo para efeito favorável na doença ${ }^{36}$.

Recentemente, outra linhagem de animal poligênico, o rato Wistar, vem sendo utilizada para estudos de obesidade induzida por dieta e tem demonstrado aumento de peso corporal ${ }^{37,38}$. Por outro lado, resultados sobre alterações na insulinemia são conflitantes. Alguns animais desenvolvem o quadro de hiperinsulinemia39, enquanto outros, não ${ }^{40}$. Avaliação de intolerância à glicose também é pouco reportada ${ }^{37}$. O modelo de rato Wistar fêmea vem sendo utilizado também como modelo DIO durante a gestação, tanto para desenvolvimento de modelo de obesidade gestacional, quanto para avaliação da prole proveniente de mãe obesa. O uso de dieta hipercalórica durante o período gestacional e de lactação de ratas Wistar parece alterar o fenótipo de obesidade nos filhos de mães obesas, demonstrando a importância da nutrição materna ${ }^{38}$.

\section{Outros modelos de roedores}

O desenvolvimento do DM2 pode ser realizado com a utilização de estreptozotocina, como no caso do modelo high-fat diet-fed streptozotocin-treated (HFD/STZ). Nesse caso, um animal DIO hiperinsulinêmico e resistente à insulina, recebe a toxina que tem a função de destruir as células-B e promover o DM2. Juntos, os dois fatores imitam a patogenia do DM2 como em humanos ${ }^{41}$. Discussões têm ocorrido com este modelo, já que a estreptozotocina é um agente utilizado para indução do diabetes tipo 1, em que há falha na produção de insulina. Em humanos, após a ocorrência do diabetes, tanto do tipo 1 quanto do tipo 2, células- $\beta$ residuais continuam existindo, porém, há diferença na quantidade dessas células entre os tipos de diabetes, com o tipo 1 apresentando menor número. A indução com a toxina normalmente afeta as células- $\beta$ de maneira grave, o que poderia promover diabetes precoce no modelo obeso e contrastar com a patogênese natural ${ }^{41}$.

\section{Modelos de roedores na cirurgia bariátrica}

Roedores também são utilizados para estudo de aspectos metabólicos da cirurgia, sendo os mais empregados os animais do tipo SD, ZFD, Zucker e $\mathrm{GK}^{42}$ e, mais recentemente, o rato Wistar, principalmente para a técnica de gastrectomia vertical $^{43}$. O trato gastrointestinal de roedores é similar ao do humano, porém, cuidados devem ser tomados, já que a pequena dimensão do animal exige maior precisão do cirurgião, assim como, instrumentos de microcirurgia. Além disso, quando se trabalha com modelos cirúrgicos de roedores, geralmente se preconiza utilizar dois grupos controles de operação simulada, com um deles recebendo a mesma quantidade de alimento que o grupo alvo de estudo (pair-fed) ${ }^{42}$.

\section{Outros modelos animais}

Além dos roedores, outras espécies podem ser utilizadas em estudos metabólicos, entre elas, os primatas não humanos. Estes animais são particularmente úteis para o estudo da obesidade e síndrome metabólica, pois a obesidade em macacos tende a ocorrer tardiamente decorrente principalmente de excessos alimentares. Além disso, o excesso de peso está comumente associado à 
alterações metabólicas semelhantes às que ocorrem na síndrome metabólica em humanos, tais como obesidade abdominal, hiperinsulinemia, intolerância à glicose e aumento dos níveis de triglicerídeos e colesterol ${ }^{1,4}$.

Animais domésticos, como cães e gatos, também podem ser utilizados, principalmente por compartilharem os mesmos fatores ambientais de risco que o ser humano, como a inatividade física, além da própria alimentação inadequada. Gatos domésticos obesos tendem a desenvolver resistência à insulina e DM2, semelhante aos humanos. Esses animais também apresentam período de prédiabetes prolongado, caracterizado por resistência à insulina e desenvolvem neuropatia e retinopatia, além de amiloidose pancreática, hipertensão e dislipidemia. É bem provável que o DM2 nessa espécie também seja uma doença poligênica, similar aos humanos, porém, a investigação de fatores genéticos em felinos ainda está iniciando ${ }^{44}$.

Apesar de modelos caninos serem relativamente bem utilizados na área de doenças metabólicas ${ }^{45}$, a patogênese das comorbidades associadas à obesidade difere do humano e é menos compreendida nesta espécie. Algumas raças possuem maior predisposição ao desenvolvimento do diabetes, principalmente o tipo 1, enquanto outras, menos, e esta doença está normalmente associada à doenças como a pancreatite', ao contrário do ser humano. Estes animais compensam de melhor forma a hiperglicemia que outras espécies, não perdendo células- $\beta$ e não apresentando amiloidose pancreática ${ }^{1,44}$. A resistência à insulina pode ocorrer, porém, o quadro total de DM2 é muito raro. Cães também apresentam hipertrigliceridemia e hipercolesterolemia, porém, são extremamente resistentes à aterosclerose. Essas constatações levam a crer que esses animais possuem mecanismos de proteção que não existem em humanos, ou então, que estes apresentam elementos fisiopatológicos para síndrome metabólica inexistentes em cães ${ }^{46}$.
Suínos são considerados bons modelos para obesidade e síndrome metabólica por sua dieta, propensão ao excesso de peso, anatomia cardiovascular e metabolismo de lipoproteínas comparáveis à humana ${ }^{47}$. Ao contrário de cães, que não desenvolvem doença vascular, suínos apresentam lesões ateroscleróticas com características anatômicas e histopatológicas similares ao que ocorre em humanos, sendo muito utilizados para este tipo de estudo. A grande desvantagem da utilização destes animais, no entanto, ocorre pelo tamanho da espécie, assim como, pelos altos custos de manutenção. Além disso, estes animais precisam de um período de ao menos dois anos para que ocorra a formação de placas ateroscleróticas, prolongando o tempo de experimento e tornando o mesmo mais oneroso ${ }^{1}$.

Devido ao seu tamanho e à similaridade anatômica com o ser humano, suínos também são empregados para procedimentos cirúrgicos. Apesar disso, algumas diferenças anatômicas no trato gastrointestinal existem, entre elas, a presença de ceco desenvolvido e extensão intestinal pronunciadamente longa. Ao contrário dos roedores, suínos são mais utilizados para aperfeiçoamento da técnica bariátrica por laparoscopia e não para o entendimento da fisiopatologia da doença, porém, o acompanhamento pós-cirúrgico dessa espécie também demonstrou perda de peso e alteração de determinadas incretinas, tanto na técnica de desvio gástrico laparoscópico em $Y$ de Roux quanto na gastrectomia vertical ${ }^{42}$.

Minipigs também podem ser utilizados, com a vantagem de serem menores que os suínos convencionais. Determinadas linhagens tendem a ser utilizadas em pesquisas metabólicas devido à facilidade de ganho de peso. Esses animais precisam ficar em restrição alimentar para manter fenótipo magro. Quando ingerem ração normal ad libitum apresentam comportamento hiperfágico, pesando de duas a três vezes mais que animais em restrição. 
A obesidade demonstrada por esta linhagem é grave e seu comportamento parece ser semelhante ao comportamento de pessoas insaciáveis, com desejo por comida. Apesar disso, é um modelo novo, não totalmente caracterizado e, embora desenvolva resistência à insulina, até o momento não houve desenvolvimento de $\mathrm{DM}^{2}$.

Recentemente, peixes-zebra têm se mostrado um modelo atrativo para estudos em doenças metabólicas, pela biologia de seu tecido adiposo, metabolismo lipídico, estrutura pancreática e homeostase da glicose ${ }^{48}$. Este vertebrado é tradicionalmente utilizado para estudos de desenvolvimento biológico por ser de custo acessível e manejo fácil, além de permitir alterações genéticas ${ }^{49}$. O primeiro modelo de peixe-zebra DIO foi relatado em 2010, com indução de dieta hipercalórica por período de oito semanas. Os indivíduos exibiram aumento do índice de massa corporal, hipertrigliceridemia e esteatose hepática quando comparados a peixes com alimentação normal ${ }^{50}$. Além disso, a análise comparativa do transcriptoma do tecido adiposo visceral destes animais demonstrou que o metabolismo lipídico deste peixe é semelhante ao dos mamíferos ${ }^{48}$. A alteração da obesidade pode ocorrer ainda por manipulação genética ${ }^{51}$ ou mesmo por substâncias modificadoras de fenótipo, como o extrato de chá verde, que inibiu o acúmulo de lipídios e alterou a expressão de genes do catabolismo lipídico ${ }^{52}$.

Da mesma maneira, indução de DM2 pode ser realizada neste modelo, imergindo o animal em soluções concentradas de glicose ${ }^{53}$. Este método, apesar de conveniente, não mimetiza a indução do diabetes em humanos. A superalimentação destes animais, entretanto, causa resistência à insulina, elevação da glicemia em jejum e intolerância à glicose ${ }^{54}$.

\section{CONSIDERAÇÕES FINAIS}

Vários modelos animais podem ser utilizados para estudo da fisiopatologia e tratamento da obesidade e síndrome metabólica, porém a descrição desses modelos ainda está longe de ser exaustiva, já que nenhum deles foi aceito como ideal para o estudo destas afecções como um todo. Animais monogênicos são a melhor escolha se um único aspecto está sendo avaliado. Já modelos DIO tendem a demonstrar melhor a interação da doença com o ambiente e o gene, porém, ainda não são totalmente efetivos para o entendimento destes distúrbios. Dessa forma, nem sempre os resultados encontrados com os modelos irão levar a novos tratamentos válidos em humanos. A procura por modelos que apresentem obesidade e síndrome metabólica da mesma forma que humanos pode ajudar a entender não só a fisiopatologia dessas afecções, mas permitir o desenvolvimento de tratamentos mais eficazes. Dessa forma é provável que novos modelos ainda sejam desenvolvidos tentando suprir essa semelhança.

O uso de animais em pesquisa deve respeitar os princípios éticos e a redução do seu número deve ocorrer sempre que possível. O aperfeiçoamento de um modelo específico e válido, potencialmente reduzirá o número de animais, assim como, o número de estudos com eles e, portanto, deve ser incentivado.

\section{A B S T R A C T}

Knowledge about animal models for metabolic study is the basis of research in this area. This work aims to review the main animal models used in the study of obesity and metabolic syndrome. For this, we performed a search in the Pubmed database using the terms "animal models", "obesity", "metabolic syndrome" and "bariatric surgery". Several species of animals can be used for the study of metabolic disorders. However, rodents are the most commonly used, both as monogenic models and as diet-induced obesity (DIO) ones. Monogenic animals are the best choice if only one aspect is being evaluated. DIO animals tend to better demonstrate the interaction between disease, environment and genetics. However, they are still not fully effective in providing understanding of all disease mechanisms.

Keywords: Models. Animal. Obesity. Metabolic Syndrome. Bariatric Surgery. 


\section{REFERÊNCIAS}

1. Harwood HJ Jr, Listrani P, Wagner JD. Nonhuman primates and other animal models in diabetes research. J Diabetes Sci Technol. 2012;6(3):503-14.

2. Nilsson C, Raun K, Yan FF, Larsen MO, Tang-Christensen M. Laboratory animals as surrogate models of human obesity. Acta Pharmacol Sin. 2012;33(2):173-81.

3. Varga $O$, Harangi $M$, Olsson IA, Hansen AK. Contribution of animal models to the understanding of the metabolic syndrome: a systematic overview. Obes Rev. 2010;11(11):792-807.

4. Lutz TA, Woods SC. Overview of animal models of obesity. Curr Protoc Pharmacol. 2012 Sep; Chapter 5:Unit5.61.

5. Wang B, Chandrasekera PC, Pippin JJ. Leptin- and leptin receptor-deficient rodent models: relevance for human type 2 diabetes. Curr Diab Rev. 2014;10(2):131-45.

6. Srinivasan K, Ramarao P. Animal models of type 2 diabetes research: an overview. Indian J Med Res. 2007;125(3):451-72.

7. Charles River Laboratories. [Internet]. Find a research model. 2018. Disponível em: https://www.criver. com/products-services/find-model.

8. The Jackson Laboratory. [Internet]. Jax® mice and services. 2018. Disponível em: https://www.jax.org/ jax-mice-and-services.

9. Hummel KP, Dickie MM, Coleman DL. Diabetes, a new mutation in the mouse. Science. 1966;153(3740):1127-8.

10. Trayhurn P, Jones PM, McGuckin MM, Goodbody $A E$. Effects of overfeeding on energy balance and brown fat thermogenesis in obese (ob/ob) mice. Nature. 1982;295(5847):323-5.

11. Memon RA, Fuller J, Moser AH, Smith PJ, Grunfeld $C$, Feingold KR. Regulation of putative fatty acid transporters and Acyl-CoA synthetase in liver and adipose tissue in ob/ob mice. Diabetes. 1999;48(1):121-7.

12. Koteish A, Diehl AM. Animal models of steatosis. Semin Liver Dis. 2001;21(1):89-104.

13. Katsuda $Y$, Ohta $T$, Miyajima K, Kemmochi $Y$, Sasase $\mathrm{T}$, Tong $\mathrm{B}$, et al. Diabetic complications in obese type 2 diabetic rat models. Exp Anim. 2014;63(2):121-34.
14. Chua SC Jr, Chung WK, Wu-Peng XS, Zhang Y, Liu SM, Tartaglia $L$, et al. Phenotypes of mouse diabetes and rat fatty due to mutations in the OB (leptin) receptor. Science. 1996;271(5251):994-6.

15. Ramos-Rodriguez JJ, Molina-Gil S, Ortiz-Barajas O, Jimenez-Palomares M, Perdomo G, Cozar-Castellano I, et al. Central proliferation and neurogenesis is impaired in type 2 diabetes and prediabetes animal models. PloS One. 2014;9(2):e89229.

16. Watadani R, Kotoh J, Sasaki D, Someya A, Matsumoto K, Maeda A. 10-Hydroxy-2-decenoic acid, a natural product, improves hyperglycemia and insulin resistance in obese/diabetic KK-Ay mice, but does not prevent obesity. J Vet Med Sci. 2017;79(9):1596-602.

17. Stütz AM, Morrison CD, Argyropoulus G. The agoutirelated protein and its role in energy homeostasis. Peptides. 2005;26(10):1771-81.

18. Bray GA, Greenway FL. Current and potential drugs for treatment of obesity. Endocr Rev. 1999;20(6):805-75.

19. Phillips MS, Liu Q, Hammond HA, Dugan V, Hey PJ, Caskey CJ, et al. Leptin receptor missense mutation in the fatty Zucker rat. Nat Genet. 1996;13(1):18-9.

20. Aleixandre de Artiñano A, Miguel Castro $M$. Experimental rat models to study the metabolic syndrome. Br J Nutr. 2009;102(9):1246-53.

21. Szayna M, Doyle ME, Betkey JA, Holloway HW, Spencer RG, Greig NH, et al. Exendin-4 decelerates food intake, weight gain, and fat deposition in Zucker rats. Endocrinology. 2000;141(6):1936-41.

22. Ramarao P, Kaul CL. Insulin resistance: current therapeutic approaches. Drugs Today (Barc). 1999;35(12):895-911.

23. Clark JB, Palmer CJ, Shaw WN. The diabetic Zucker fatty rat. Proc Soc Exp Biol Med. 1983;173(1):68-75.

24. Yasuda K, Nishikawa W, Iwanaka N, Nakamura E, Seino $Y$, Tsuda $K$, et al. Abnormality in fibre type distribution of soleus and plantaris muscles in non-obese diabetic Goto-Kakizaki rats. Clin Exp Pharmacol Physiol. 2002;29(11):1001-8.

25. Portha B, Giroix $M H$, Serradas $P$, Gangneurau MN, Movassat J, Rajas F, et al. beta-cell function and viability in the spontaneously diabetic GK rat: information from the GK/Par colony. Diabetes. 2001;50 Suppl1:S89-93. 
26. Joost HG. The genetic basis of obesity and type 2 diabetes: lessons from the New Zealand obese mouse, a polygenic model of the metabolic syndrome. Results Probl Cell Differ. 2010;52:1-11.

27. Cho YR, Kim HJ, Park SY, Ko HJ, Hong EG, Higashimori T, et al. Hyperglycemia, maturity-onset obesity, and insulin resistance in NONcNZO10/LtJ males, a new mouse model of type 2 diabetes. Am J Physiol Endocrinol Metab. 2007;293(1):E327-36.

28. Russell JC, Proctor SD. Small animal models of cardiovascular disease: tools for the study of the roles of metabolic syndrome, dyslipidemia, and atherosclerosis. Cardiovasc Pathol. 2006;15(6):318-30.

29. Madsen AN, Hansen G, Paulsen SJ, Lykkegaard K, Tang-Christensen M, Hansen HS, et al. Longterm characterization of the diet-induced obese and diet-resistant rat model: a polygenetic rat model mimicking the human obesity syndrome. J Endocrinol. 2010;206(3):287-96.

30. Sato Mito N, Suzui M, Yoshino H, Kaburagi T, Sato $K$. Long term effects of high fat and sucrose diets on obesity and lymphocyte proliferation in mice. J Nutr Health Aging. 2009;13(7):602-6.

31. Aydin S, Aksoy A, Aydin S, Kalayci M, Yilmaz M, Kuloglu T, et al. Today's and yesterday's of pathophysiology: biochemistry of metabolic syndrome and animal models. Nutrition. 2014;30(1):1-9.

32. Basumata C, Kalita JC, Dutta C, Mohan P, Baruah KK. Animal models for type 2 diabetes. Int J Int Sci Inn Tech Sec B. 2012;1(3):24-30.

33. Sheludiakova A, Rooney K, Boakes RA. Metabolic and behavioural effects of sucrose and fructose/glucose drinks in the rat. Eur J Nutr. 2012;51(4):445-54.

34. Surwit RS, Feinglos MN, Rodin J, Sutherland A, Petro AE, Opara EC, et al. Differential effects of fat and sucrose on the development of obesity and diabetes C57BL/6J and AJJ mice. Metabolism. 1995;44(5):645-51.

35. Oron-Herman M, Kamari $Y$, Grossman E, Yeger G, Peleg E, Shabtay Z, et al. Metabolic syndrome: comparison of the two commonly used animal models. Am J Hypertens. 2008;21(9):1018-22.

36. Verbeek J, Lannoo M, Pirinen E, Ryu D, Spincemaille P, Vander Elst I, et al. Roux-en-y gastric bypass attenuates hepatic mitochondrial dysfunction in mice with nonalcoholic steatohepatitis. Gut. 2015;64(4):673-83.
37. Da Silva AS, Pauli JR, Ropelle ER, Oliveira AG, Cintra $D E$, De Souza CT, et al. Exercise intensity, inflammatory signaling, and insulin resistance in obese rats. Med Sci Sports Exerc. 2010;42(12):2180-8.

38. Kimura Y, Yamada A, Takabayashi Y, Tsubota T, Kasuga $H$. Development of a new diet-induced obesity (DIO) model using Wistar lean rats. Exp Anim. 2018;67(2):155-61.

39. Nascimento AF, Sugizaki MM, Leopoldo AS, LimaLeopoldo AP, Luvizotto RA, Nogueira CR, et al. A hypercaloric pellet-diet cycle induces obesity and co-morbidities in Wistar rats. Arq Bras Endocrinol Metabol. 2008;52(6):968-74.

40. Estadella D, Oyama LM, Dâmaso AR, Ribeiro EB, Oller do Nascimento CM. Effect of palatable hyperlipidic diet on lipid metabolism of sedentary and exercised rats. Nutrition. 2004;20(2):218-24.

41. Skovsø S. Modeling type 2 diabetes in rats using high fat diet and streptozotocin. J Diabetes Invest. 2014;5(4):349-58.

42. Rao RS, Rao V, Kini S. Animal models in bariatric surgery-a review of the surgical techniques and postsurgical physiology. Obes Surg. 2010;20(9):1293-305.

43. Rodríguez $A$, Becerril $S$, Valentí $V$, Moncada $R$, Méndez-Giménez $L$, Ramírez $B$, et al. Short-term effects of sleeve gastrectomy and caloric restriction on blood pressure in diet-induced obese rats. Obes Surg. 2012;22(9):1481-90.

44. Nelson RW, Reusch CE. Animal models of disease: classification and etiology of diabetes in dogs and cats. J Endocrinol. 2014;222(3):T1-9.

45. Bergman RN, Kim SP, Hsu IR, Catalano KJ, Chiu $J D$, Kabir $M$, et al. Abdominal obesity: role in the pathophysiology of metabolic disease and cardiovascular risk. Am J Med. 2007;120(2 Suppl 1):S3-8.

46. Verkest KR. Is the metabolic syndrome a usefull clinical concept in dogs? A review of the evidence. Vet J. 2014;199(1):24-30.

47. Hamamdzic D, Wilensky RL. Porcine models of accelerated coronary atherosclerosis: role of diabetes mellitus and hypercholesterolemia. J Diabetes Res. 2013;2013:761415.

48. Zang L, Maddison LA, Chen W. Zebrafish as a model for obesity and diabetes. Front Cell Dev Biol. 2018;6:91. 
49. Freifeld L, Odstrcil I, Förster D, Ramirez A, Gagnon JA, Randlett O, et al. Expansion microscopy of zebrafish for neuroscience and developmental biology studies. Proc Natl Acad Sci U S A. 2017;114(50):E10799-E10808.

50. Oka T, Nishimura $Y$, Zang L, Hirano M, Shimada $Y$, Wang $Z$, et al. Diet-induced obesity in zebrafish shares common pathophysiological pathways with mammalian obesity. BMC Physiol. 2010;10:2.

51. McMenamin SK, Minchin JE, Gordon TN, Rawls JF, Parichy DM. Dwarfism and increased adiposity in the gh1 mutant zebrafish vizzini. Endocrinology. 2013;154(4):1476-87.

52. Meguro S, Hasumura T, Hase T. Body fat accumulation in zebrafish is induced by a diet rich in fat and reduced by supplementation with green tea extract. PloS One. 2015;10(3):e0120142.
53. Capiotti KM, Antonioli R Jr, Kist LW, Bogo MR, Bonan CD, Da Silva RS. Persistent impaired glucose metabolism in a zebrafish hyperglycemia model. Comp Biochem Physiol B Biochem Mol Biol. 2014;171:58-65

54. Zang L, Shimada Y, Nishimura N. Development of a novel zebrafish model for type 2 diabetes mellitus. Sci Rep. 2017;7(1):1461.

Recebido em: 07/08/2018

Aceito para publicação em: 20/09/2018

Conflito de interesse: nenhum.

Fonte de financiamento: nenhuma.

Endereço para correspondência:

Taíse Fuchs

E-mail: taisefuchs@hotmail.com taise.fuchs@up.edu.br

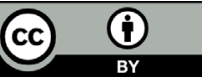

